\title{
Lipopeptides derived from BP100 containing a D-amino acid or a His residue
}

\author{
Angel Oliveras, Esther Badosa, Emilio Montesinos, Lidia Feliu, Marta Planas
}

University of Girona, Spain

https://doi.org/10.17952/35EPS.2018.233

\section{Introduction}

Plant diseases caused by bacteria and fungi are currently one of the major problems in agriculture, producing important economic losses. The solution to this problem relies on the use of copper, antibiotics or fungicides. These compounds are efficient; however, they are regarded as serious environmental contaminants. Moreover, antibiotics are not allowed in many countries and their use is hampered by the appearance of resistant strains [1].

Antimicrobial peptides have emerged as a good alternative to traditional pesticides. They display a broad spectrum of activity and do not easily facilitate the development of microbial resistance [2]. In this context, we have identified the peptide H-Lys-Lys-Leu-Phe-Lys-Lys-Ile-Leu-Lys-Tyr-Leu- $\mathrm{NH}_{2}$ (BP100), which displays high antibacterial activity in vitro and low hemolysis [3].

The acylation of antimicrobial peptides is a strategy to increase their biological activity and their stability to protease degradation [4]. Based on these considerations, a collection of lipopeptides derived from BP100, incorporating an acyl chain (butanoyl, hexanoyl or lauroyl group) at the N-terminus or at the side-chain of each residue of the sequence, was synthesized [5]. Lipopeptides with an interesting biological activity profile were identified.

On the other hand, the incorporation of one or more D-amino acids into the BP100 sequence was evaluated. BP143, that incorporates a D-Phe at position four, showed a similar antibacterial activity to that of BP100 but it was less hemolytic and more stable to protease degradation [6].

Herein, with the aim of improving the hemolysis and the stability to protease degradation of the lipopeptides derived from BP100, 21 new lipopeptides containing one or two D-amino acids or a histidine residue were designed, synthesized and evaluated for their biological activity.

\section{Results and Discussion}

Lipopeptides were synthesized on solid phase as C-terminal amides following a standard 9-fluorenylmethoxycarbonyl (Fmoc)/tert-butyl (tBu) strategy using Fmoc-Rink-MBHA as solid support. To obtain the lipopeptides incorporating the acyl group at the side-chain of a Lys residue, this amino acid was incorporated as Fmoc-Lys(ivDde)-OH. All lipopeptides were purified, characterized by HPLC and HRMS and obtained in excellent purities ( $>99 \%)$.

Peptides were tested for their in vitro antibacterial activity. The minimum inhibitory concentration(MIC) was determined. In general, the lipopeptides containing one D-amino acid at position four were as active as the corresponding parent lipopeptide against all the phytopathogens assayed. On the other hand, lipopeptides containing a His residue or two D-amino acids were less active than their corresponding counterparts.

The toxicity of antimicrobial peptides targeting the bacterial membrane can be assessed with animal cell model systems, being erythrocytes the most commonly used. Hemolysis was determined from erythrocyte suspensions of horse blood at a lipopeptide concentration of $250 \mu \mathrm{M}$. The introduction of one or two D-amino acids or a His residue in lipopeptides containing a butanoyl or a hexanoyl group resulted in a decrease of the hemolysis. In contrast, no improvement was observed for peptides incorporating a lauroyl group.

The phytotoxicity was evaluated for their effect upon infiltration on tobacco leaves as described previously [7]. All lipopeptides containing a D-amino acid or a His residue were less hemolytic than melittin, which was used as reference product. However, no general trend was observed when comparing the phytotoxicity of these lipopeptides with that of the parent lipopeptides.

Lipopeptides with the best biological activity profile were D-BP387 and D-BP389 that displayed MIC values between 0.7 and $12.5 \mu \mathrm{M}$ against all eight phytopathogens, they were not hemolytic and were less phytotoxic than melittin (Table 1). 
Table 1: MIC values, hemolysis and phytotoxicity of the lipopeptides with the best biological activity profile

\begin{tabular}{|c|c|c|c|c|c|c|c|c|c|c|}
\hline \multirow{2}{*}{ Lipopeptide } & \multicolumn{8}{|c|}{$\operatorname{MIC}(\mu \mathrm{M})$} & \multirow{2}{*}{$\begin{array}{c}\mathbf{H}^{\mathrm{b}} \\
(\%)\end{array}$} & \multirow{2}{*}{$\begin{array}{r}\text { Phyt }^{\circ} \\
(\%)\end{array}$} \\
\hline & $E a^{\mathrm{a}}$ & $P s s^{\mathrm{a}}$ & $X a p^{\mathrm{a}}$ & $X f a$ & $P s a^{\mathrm{a}}$ & $X a v^{\mathrm{a}}$ & $P e^{\mathrm{a}}$ & $F O^{\mathrm{a}}$ & & \\
\hline BP387 & $3.1-6.2$ & $3-1-6.2$ & $3.1-6.2$ & $1.6-3.1$ & $3.1-6.2-$ & $1.6-3.1$ & $6.2-12.5$ & $0.8-1.6$ & 14 & 9 \\
\hline D-BP387 & $6.2-12.5$ & $3.1-6.2$ & $3.1-6.2$ & $6.2-12.5$ & $1.6-3.1$ & $3.1-6.2$ & $3.1-6.2$ & $0.8-1.6$ & 0 & 4 \\
\hline BP389 & $3.1-6.2$ & $6.2-12.5$ & $0.8-1.6$ & $1.6-3.1$ & $3.1-6.2$ & $0.8-1.6$ & $6.2-12.5$ & $1.6-3.1$ & 22 & 9 \\
\hline D-BP389 & $3.1-6.2$ & $3.1-6.2$ & $0.8-1.6$ & $1.6-3.1$ & $1.6-3.1$ & $1.6-3.1$ & $3.1-6.2$ & $0.8-1.6$ & 0 & 5 \\
\hline
\end{tabular}

a) Ea, Erwinia amylovora; Pss, Pseudomonas syringae pv. syringae; Xap, Xanthomonas arboricola pv. pruni; $\mathrm{Xf}$, Xanthomonas fragariae; Psa, Pseudomonas syringae pv. actinidiae; Xav, Xanthomonas axonopodis pv. vesicatoria; Pe, Penicillium expansum; Fo, Fusarium oxysporum.

b) $\mathrm{H}$, Percentage of hemolysis at $250 \mu \mathrm{M}$.

c) Phyt, Percentage of phytotoxicity at $250 \mu \mathrm{M}$

\section{References}

1 Agrios, GN. Plant Pathology, 5th ed. Academic Press: San Diego, California, 2005.

2 Baltzer, SA; Brown, MH. Antimicrobial Peptides - Promising Alternatives to Conventional Antibiotics. J. Mol. Microbiol. Biotechnol. 2011; 20: 228-235; DOI:10.1159/000331009.

3 Badosa, E; Ferre, R; Planas, M; Feliu, L; Besalú, E; Cabrefiga, J; Bardají, E; Montesinos, E. A Library of Linear Undecapeptides with Bactericidal Activity against Phytopathogenic Bacteria. Peptides 2007; 28: 2276-2285; DOI:10.1016/j.peptides.2007.09.010.

4 Vilà, S; Badosa, E; Montesinos, E; Planas, M; Feliu, L. Synthetic Cyclolipopeptides Selective against Microbial, Plant and Animal Cell Targets by Incorporationof D-Amino Acids or Histidine. PLoS One 2016; 11: e0151639; DOI:10.1371/journal.pone.0151639.

5 Oliveras, À; Baro, A; Montesinos, L; Badosa, E; Montesinos, E; Feliu, L; Planas, M. Antimicrobial Activity of Linear Lipopeptides Derived from BP100 towards Plant Pathogens. PLoS One 2018; 13: 1-17; DOI:10.1371/journal.pone.0201571.

6 Güell, I; Cabrefiga, J; Badosa, E; Ferre, R; Talleda, M; Bardají, E; Planas, M; Feliu, L; Montesinos, E. Improvement of the Efficacy of Linear Undecapeptides against Plant-Pathogenic Bacteria by Incorporationof D-Amino Acids. Appl. Environ. Microbiol. 2011; 77: 2667-2675; DOI:10.1128/AEM.02759-10.

7 Badosa, E; Moiset, G; Montesinos, L; Talleda, M; Bardají, E; Feliu, L; Planas, M; Montesinos, E. Derivatives of the Antimicrobial Peptide BP100 for Expression in Plant Systems. PLoS One 2013; 8: 1-12; DOI:10.1371/journal.pone.0085515. 